\title{
PACT: the actuator to support the primary mirror of the ELT
}

\author{
F. Kamphues ${ }^{\mathrm{a}}$, J. Nijenhuis ${ }^{\mathrm{a}}$, R. den Breeje ${ }^{\mathrm{a}}$, T.C. van den Dool ${ }^{\mathrm{a}}, \mathrm{J}$. Ponsioen ${ }^{\mathrm{a}}$ \\ ${ }^{\mathrm{a}}$ TNO Science \& Industry, Stieltjesweg 1, $2628 \mathrm{CK}$, Delft, The Netherlands
}

\begin{abstract}
The European Southern Observatory (ESO) has started technology development for their next generation optical telescope. Due to its ultra large collecting area, The European Extremely Large Telescope (E-ELT) will require a paradigm shift in telescope design to keep the overall program cost at an acceptable level. The E-ELT will feature a 42 meter segmented primary mirror and will make extensive use of active and adaptive optics. Each primary mirror segment will be supported by three actuators that control piston and tilt. TNO has developed a low cost nanopositioning actuator (PACT) for the primary mirror segments. The actuators will be tested by IAC and ESO, with support from TNO, under operational conditions in a Wind Evaluation Breadboard (WEB) at the Roque de Los Muchachos observatory in La Palma.
\end{abstract}

Keywords: low cost nano actuator, nano positioning, large segmented mirror, E-ELT, TMT, telescope.

\section{INTRODUCTION}

In December 2006, ESO announced the start of a detailed design study for the European Extremely Large Telescope, (EELT, see Figure 1). E-ELT will have a segmented primary mirror with a diameter of $42 \mathrm{~m}$. Limitations in manufacturing and transport methods require the use of a segmented primary mirror. The concept of a segmented primary mirror is successfully used on the Keck telescopes in Hawaii. In recent years more telescopes with segmented primary mirrors have seen first light: The HET, SALT and GTC telescopes all use segmented primary mirrors.

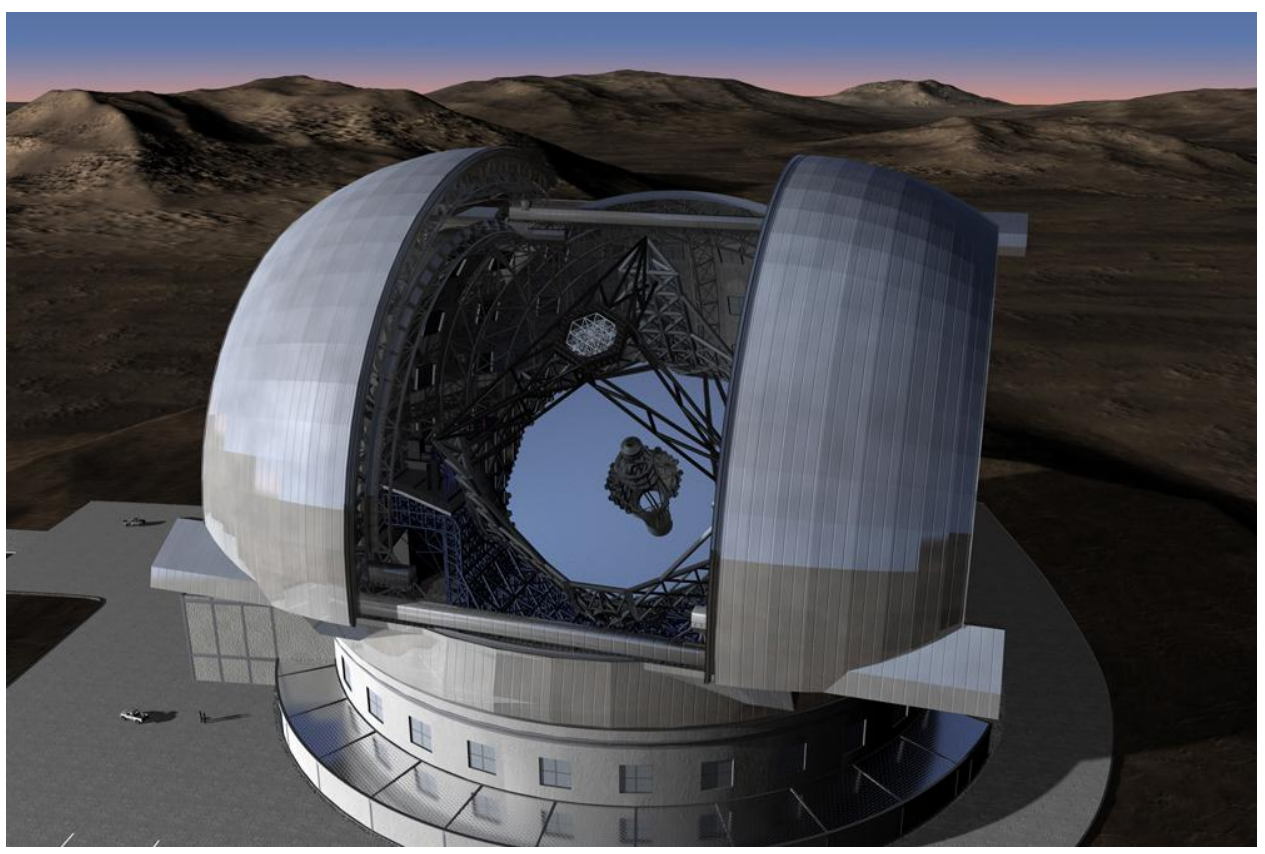

Figure 1 Artist impression of the E-ELT (courtesy of ESO)

ben.braam@tno.nl; phone +31(0)152692180; fax +31(0)152692111; www.tno.nl

Advanced Optical and Mechanical Technologies in Telescopes and Instrumentation, edited by Eli Atad-Ettedgui, Dietrich Lemke, Proc. of SPIE Vol. 7018, 70180Z, (2008) · 0277-786X/08/\$18 · doi: 10.1117/12.789921 
The E-ELT primary mirror will be composed of approximately 1000 hexagonal segments, that fit in an inscribed circle of $\varnothing 1.8 \mathrm{~m}$. All segments of the primary mirror must be accurately co-phased to within 5 nanometers RMS. This is a daunting challenge. Not only because of the required performance under wind loading and mechanical vibration, but also because the actuators must be low cost and suitable for mass production. A typical mirror cell is shown in Figure 2.

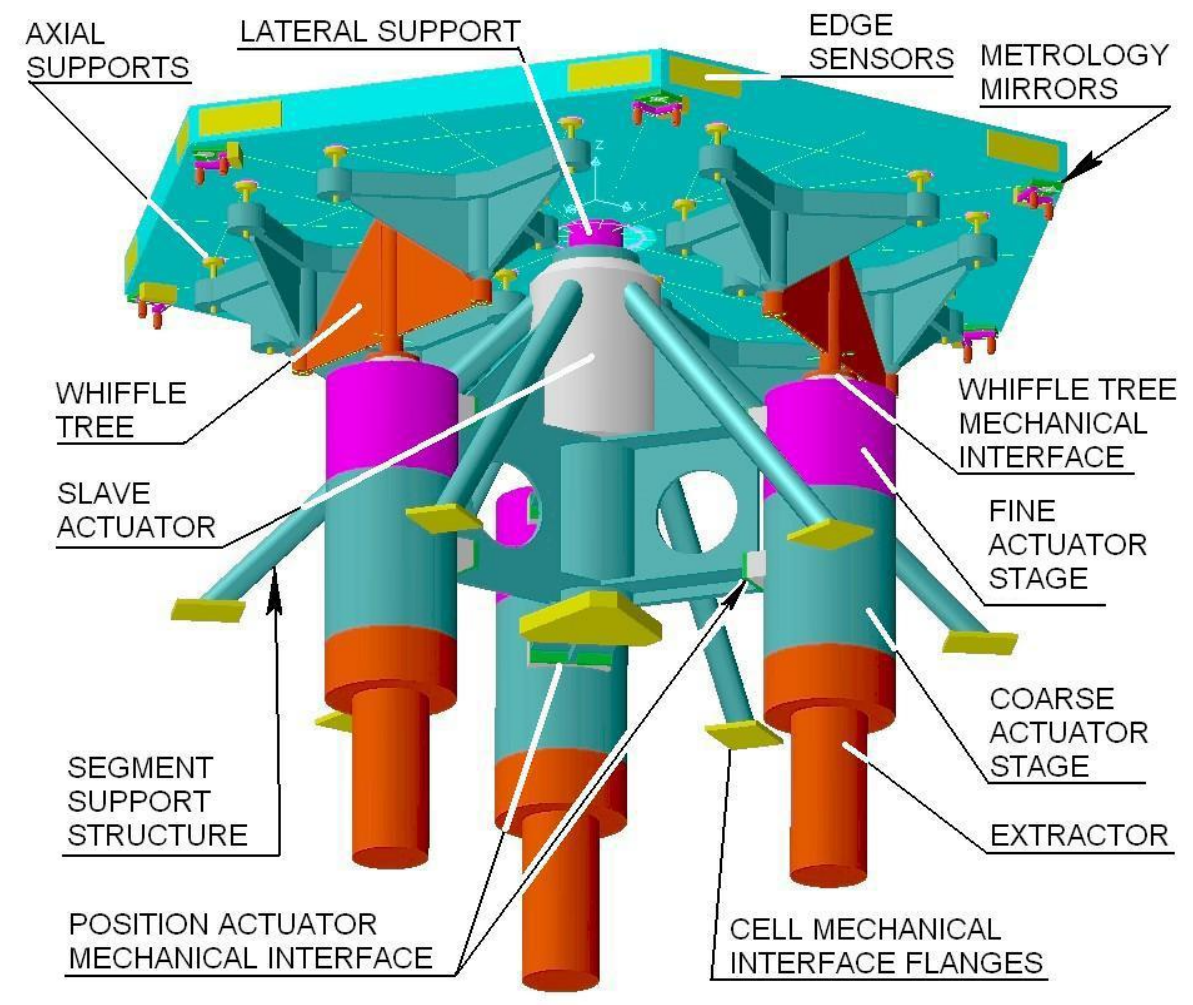

Figure 2 E-ELT mirror cell (image courtesy of ESO)

\section{REQUIREMENTS}

For E-ELT two configurations are possible:

- $\quad 510 \mathrm{~kg}$ Glass segments (including whiffle tree)

- $180 \mathrm{~kg} \mathrm{SiC}$ segments (including whiffle tree)

Although $\mathrm{SiC}$ mirrors will be much more expensive, it is expected that $\mathrm{SiC}$ mirrors will offer a price and performance benefit at system level.

The key requirements for PACT are listed in Table 1. A total of 20 actuators have been built. 18 actuators will be tested by IAC and ESO, with support from TNO. The tests will take place under operational conditions in a Wind Evaluation Breadboard (WEB) at the Roque de Los Muchachos observatory in La Palma. 
Table 1 PACT key requirements

\begin{tabular}{|c|c|}
\hline Function & Requirement \\
\hline Axial stiffness & Resonance frequency $>120 \mathrm{~Hz}$. \\
\hline Compression load & $\begin{array}{l}170 \mathrm{~kg} \text { glass ceramic segments. } \\
60 \mathrm{~kg} \mathrm{SiC} \text { segments }\end{array}$ \\
\hline Tension load & $\begin{array}{l}\text { PACT design compatible with equal tension and } \\
\text { compression loads. }\end{array}$ \\
\hline Wind load & $\begin{array}{l}25 \mathrm{~N} \text { rms per segment } \\
60 \mathrm{~N} \text { peak per segment }\end{array}$ \\
\hline Lateral load & Max. $10 \%$ of compression load \\
\hline \multirow{2}{*}{ Position accuracy } & $\begin{array}{l}\text { Course stage } \\
<50 \mu \mathrm{m}\end{array}$ \\
\hline & $\begin{array}{l}\text { Fine stage } \\
<5 \mathrm{~nm} \mathrm{rms} \mathrm{in} \mathrm{observation} \mathrm{mode}\end{array}$ \\
\hline \multirow{3}{*}{ Mechanical stroke PACT } & $\begin{array}{l}\text { Course stage } \\
15 \mathrm{~mm}(\text { goal } 30 \mathrm{~mm})\end{array}$ \\
\hline & $\begin{array}{l}\text { Fine stage } \\
>0.5 \mathrm{~mm}\end{array}$ \\
\hline & $\begin{array}{l}\text { Extractor stroke } \\
150 \mathrm{~mm}\end{array}$ \\
\hline Closed loop bandwidth & $100 \mathrm{~Hz}$ \\
\hline Maximum tracking speed & $2.5 \mu \mathrm{m} / \mathrm{s}$ \\
\hline Maximum slewing speed & $60 \mu \mathrm{m} / \mathrm{s}$ \\
\hline \multirow{2}{*}{ Power dissipation } & $\begin{array}{l}\text { Operational mode } \\
<2.0 \mathrm{~W}\end{array}$ \\
\hline & $\begin{array}{l}\text { Slewing mode } \\
<3.75 \mathrm{~W}\end{array}$ \\
\hline Mass & $<15 \mathrm{~kg}$ \\
\hline WEB PACT reliability & MTBF $>6$ months \\
\hline ELT PACT reliability & MTBF $>5$ years \\
\hline Recurring cost for E-ELT & $\begin{array}{l}<4000 \text { Euro per actuator (for a glass segment) } \\
<3500 \text { Euro per actuator (for a } \mathrm{SiC} \text { segment) }\end{array}$ \\
\hline
\end{tabular}

\section{DESIGN DESCRIPTION}

The primary function of PACT is to maintain the position of the M1 mirror of the E-ELT to within $5 \mathrm{~nm}$ rms under dynamic wind loads and gravity deflections. An extra complication is the requirement for an extractor function of 150 $\mathrm{mm}$, to be able to remove mirror elements for maintenance.

An extensive trade off study was done to determine the optimum concept for the given set of requirements. To be able to meet the positioning requirements, a two stage actuator is the most logical approach, although the Keck telescope successfully uses a single stage actuator with hydraulic reductor to achieve the required resolution [1]. The 2008 cost of the Keck actuator however would be in the order of $\$ 40.000$ and doesn't meet the E-ELT stroke requirements [2]. The use of hard or soft actuators was also considered [3, 4]. 
Examples of hard actuators are:

- Piezo stack/Piezo motor

- $\quad$ Motorized screw

- Hydraulic actuator

Examples of soft actuators are:

- Voice coil

- Linear motor

- Pneumatic actuator

Hard actuators cannot control disturbances beyond the control bandwidth. A soft actuator acts as a filter for high frequency disturbances beyond the control bandwidth and will lead to better mirror stability and prevents potentially catastrophic overload. A soft actuator allows for a lower bandwidth, which could be important given the limitations with the current generation edge sensors. A soft actuator requires some form of off-loading, due to the continuously changing axial load, caused by the diurnal motion.

The outcome of the TNO trade-off was a two stage actuator. A two stage concept allows the use of relatively inexpensive standard industrial components. A motorized screw was selected for the coarse stage. The motorized screw is also capable of providing the extractor function, thereby removing the need for an additional component. A soft actuator (voice coil) is used for the fine stage. Compression springs take care of the off-loading.

The fine stage has a stroke of $0.55 \mathrm{~mm}$, limited by the stall force of the voice coil. The coarse stage desaturates the fine stage to minimize power consumption, limiting the operational voice coil stroke to a few microns. The PACT actuator is shown in Figure 3.

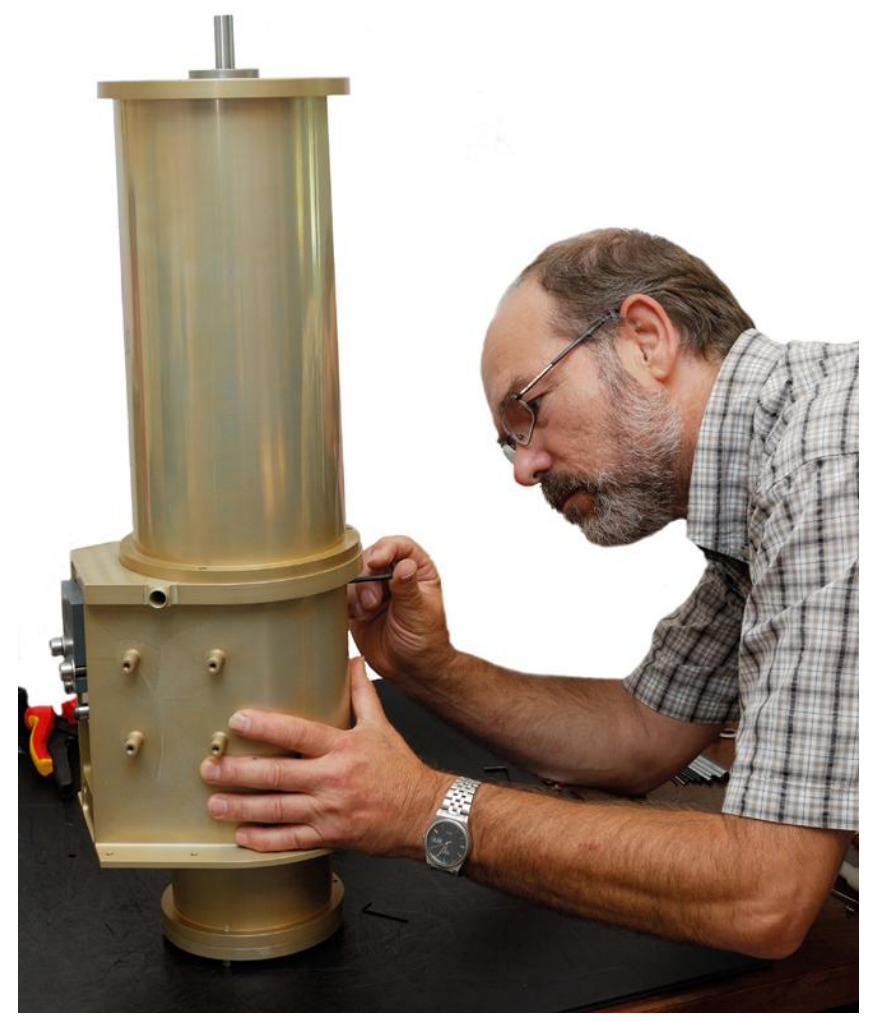

Figure 3 The PACT actuator 
Three actuators control the piston and tip/tilt of the mirror element. A separate support constrains the lateral movement of the mirror. Nevertheless several thermo-mechanical phenomena will cause that the PACT interface surface will experience a lateral and an angular motion relative to the whiffle tree. These causes can be:

- Temperature difference between whiffle tree and PACT.

- Difference in thermal expansion between mirror cell, whiffle tree and PACT.

- Mechanical deformation of the support structure of ELT due to gravity effects.

The design of PACT takes these lateral loads into account.

The PACT actuator uses a number of standard components, but makes use of TNO's extensive knowledge of precision mechanisms, nanopositioning and advanced control engineering. The PACT control system is derived from programs like the Darwin cryogenic delay line, VLTI PRIMA star separator, Darwin Nulling Breadboard, etc. The block diagram of the control system is shown in Figure 4.

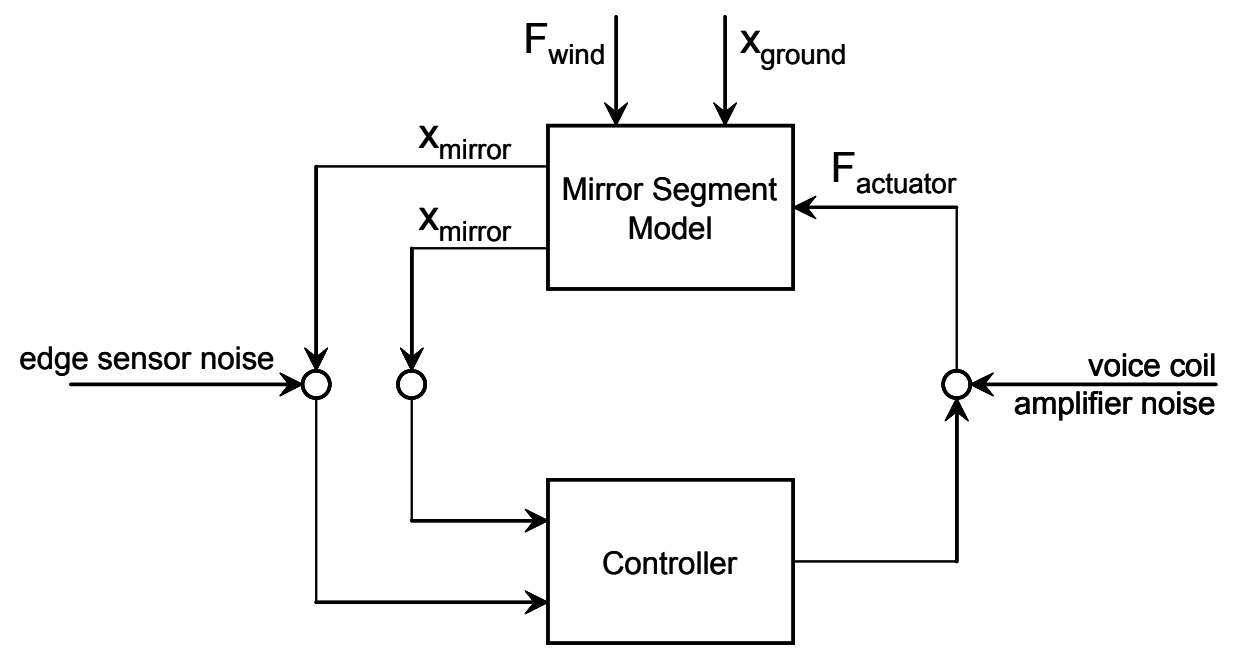

Figure 4 Control system block diagram

The performance of an actively controlled dynamic system depends on the following three components:

A dynamic model of the system to be controlled. Two models were used to predict the performance of PACT. First, a highly idealized simple model was used to explore the limits of performance. Given this model the achievable performance was determined by the disturbances and the controller. Second, a FEM model of the test-setup was used to predict the performance of PACT in the test-setup. Ideally the dynamics of PACT should have negligible influence on the achievable performance. The design is such that the first natural frequency relevant for positioning lies at above $220 \mathrm{~Hz}$.

Disturbances. Two types of disturbances are defined by ESO, namely wind disturbances and sensor noise of the edge sensors. Additional disturbances (e.g. voice coil amplifier noise) were also taken into account.

Controller. An optimal controller was calculated for the ideal system to explore the upper limit of achievable performance. For the FEM model a controller was calculated to show that the predicted performance of PACT meets the requirements. 
The temporal spectrum of the wind loading disturbance for a single mirror segment is shown in Figure 5. The ESO spectrum has essentially an infinite order. To make the design and analysis of (optimal) controllers more tractable we have defined a second order approximate filter that acts as an upper bound for the "true"spectrum up until $200 \mathrm{~Hz}$. The mirror mass and the wind loading have been scaled down to the values for a single actuator (170 kg Zerodur mirror and $10 \mathrm{~N}$ wind loading rms). The approximate filter for the disturbance level for a single actuator has the following transfer function.

$$
W_{\text {wind }}(s)=\frac{459.2}{(s+2 \pi \cdot 0.25)(s+2 \pi \cdot 4)}
$$

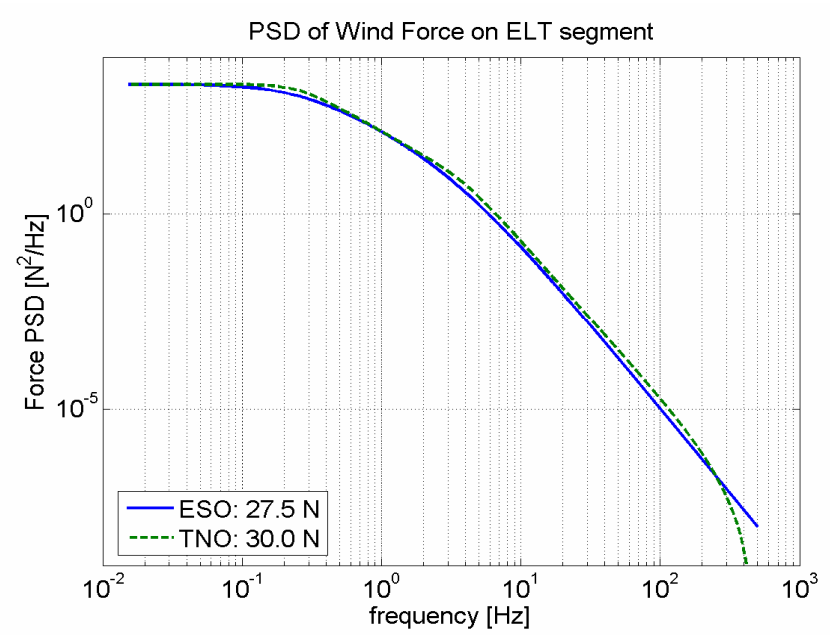

Figure 5 Wind force spectrum on mirror segment

The noise of the edge sensor on WEB has a level of $0.2 \mathrm{~nm} / \sqrt{\mathrm{Hz}}$ for the piston mode and is filtered with an anti-aliasing filter as specified by ESO. Since we are only interested in the achievable performance for the given disturbance level and not in the limitations imposed by the hardware implementation of the control system, the edge sensor noise has been modelled as white noise with a noise level of $0.2 \mathrm{~nm} / \sqrt{\mathrm{Hz}}$ without additional filtering.

\section{DEVELOPMENT AND VERIFICATION PROGRAM}

In order to verify the selected concept and to validate the chosen control strategy, a breadboard actuator was built and tested (Figure 6). After successful completion of the breadboard program and demonstration of the performance, the detailed design of the PACT actuator was started.

Two prototype actuators were built and extensively tested in order to optimize the control algorithm. A dedicated test setup was used to prove that PACT meets the positioning requirements (Figure 7). The control algorithm runs on a Real Time Linux system (which offers flexibility when modifying control parameters). The PACT test setup was analyzed using FEM. The primary issue here is that natural frequency modes that influence the positioning accuracy of PACT should remain above $3 \mathrm{x}$ the control bandwidth. Present bandwidth is around $80 \mathrm{~Hz}$. Therefore the required minimum natural frequency of the structure should be around $240 \mathrm{~Hz}$. With a dummy mass of $170 \mathrm{~kg}$ this means that the structure supporting this mass and PACT must be very stiff. A typical value for this stiffness is $6.10^{8} \mathrm{~N} / \mathrm{m}$. Because of this very high stiffness requirement PACT and dummy mass are interconnected by a rectangular box. PACT is mounted on top of it while the dummy mass is inside. The dummy mass is guided by two leaf springs that are connected to the walls of the box. The test setup allows the PACT to be tilted to simulate gravity loading. The performance of the PACT prototype actuators was successfully demonstrated in the test setup. Subsequently 18 actuators for WEB were built. The same test setup was also used for acceptance testing. 


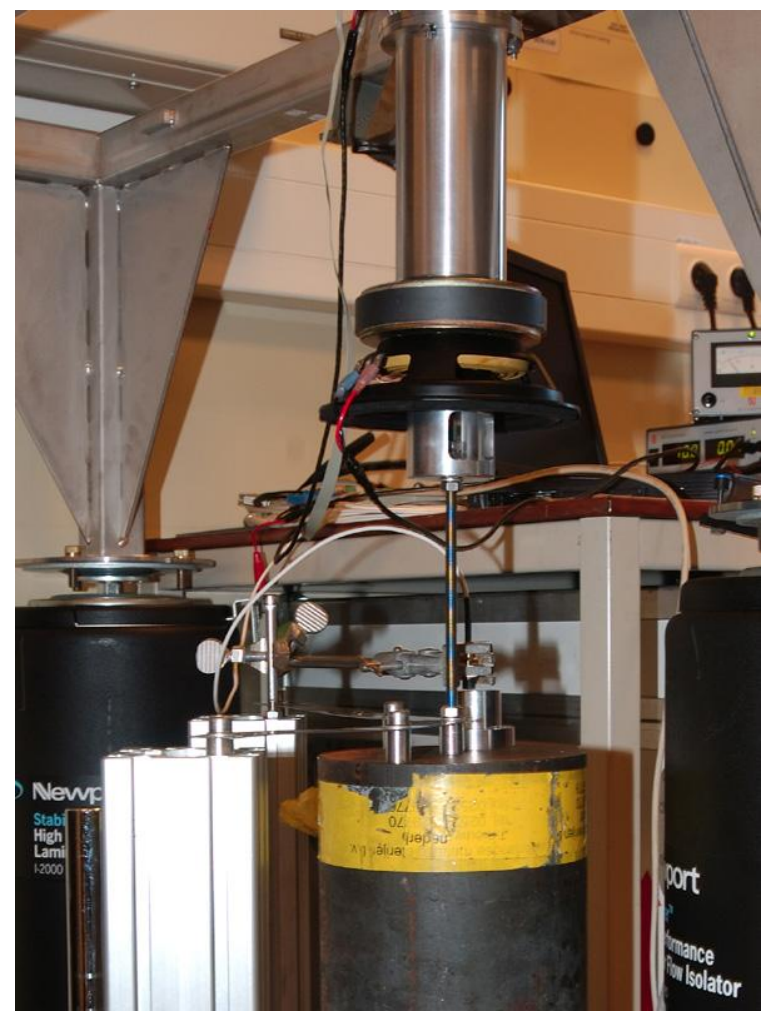

Figure 6 Breadboard actuator

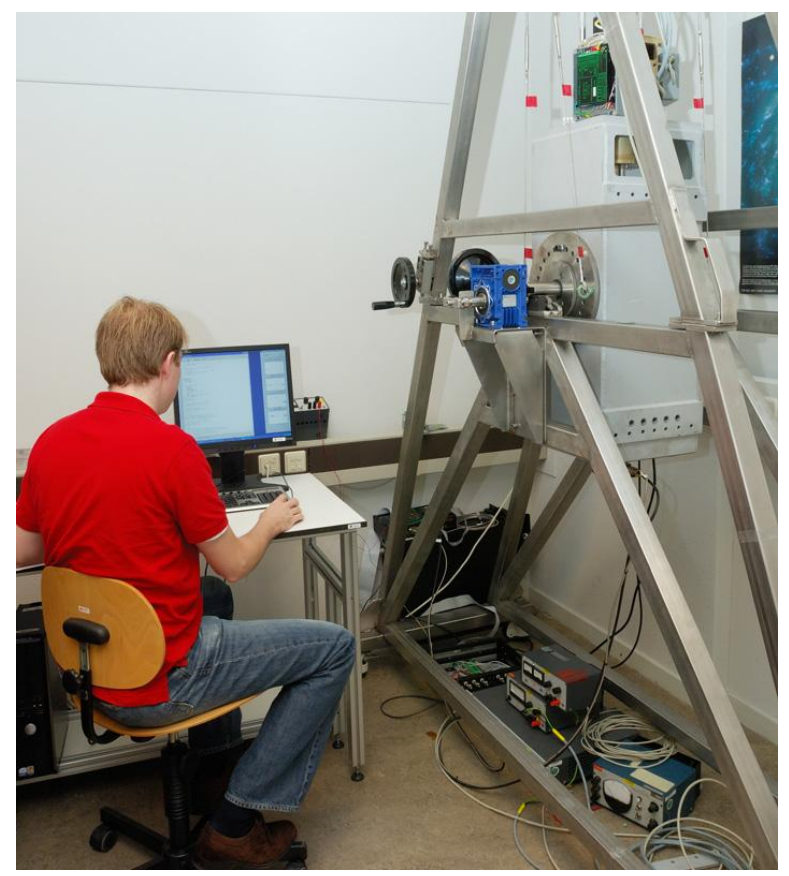

Figure 7 PACT test setup

Proc. of SPIE Vol. 7018 70180Z-7 


\section{PERFORMANCE AND RELIABILTY}

The PACT actuator meets all technical requirements, except for the power dissipation with the glass segments. For the glass segment the total power dissipation in observation mode per actuator is $3.3 \mathrm{~W}$ (specified $<2 \mathrm{Watt}$ ). This is caused by the holding current of the stepper motor. A different motor will be used for the final E-ELT actuators, if the glass segments are the selected baseline.

The position accuracy at the maximum observation speed of $2.5 \mu \mathrm{m} / \mathrm{s}$ is $2.5 \mathrm{~nm} \mathrm{rms} \mathrm{(at} 0 \mathrm{~m} / \mathrm{s}$ wind speed) and $3.7 \mathrm{~nm}$ $\mathrm{rms}$ (at $5 \mathrm{~m} / \mathrm{s}$ wind speed). Even in slewing mode (at $60 \mu \mathrm{m} / \mathrm{s}$ ), the positioning error of the actuators is very small, approximately $30 \mathrm{~nm}$ rms (Figure 8).
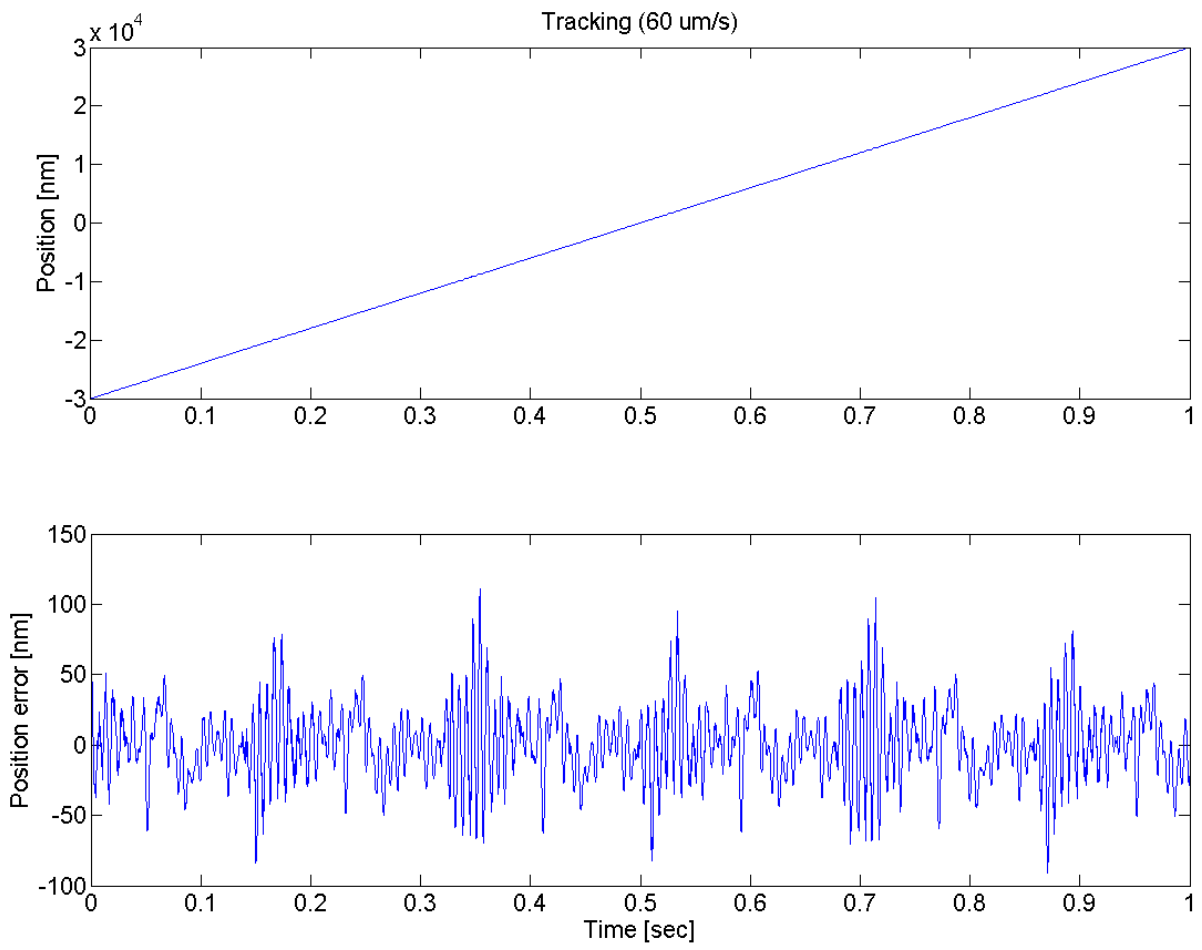

Figure 8 position error during slewing 
The actuator uses standard industrial components and can be built at relatively low cost and is suitable for mass production.

Reliability of a complex system like the E-ELT is of utmost importance for a proper science return. Due to its large number of components, the M1 control system is one of the critical items in E-ELT. Reliability starts of course with design. Several steps have been taken to increase the reliability of the actuator:

- Proper design of cabling, stress reliefs and soldered joints

- Limitation of lateral loads, which could lead to excessive wear

- Contactless voice coil

- Elastic guide mechanism with long fatigue life

- Industrial motorized screw with ample margin on life

- Low power operation of electronics ensure low temperatures and long life

- Hermetic sealing of all components to prevent the ingress of dust and moisture

- The use of a fault tolerant algorithm and prevention of catastrophic failure

For E-ELT, reliability can be further increased by adding some redundancy. Upon completion of the WEB test program, the design of the PACT will be further optimized for use in final E-ELT configuration.

\section{WIND EVALUATION BREADBOARD}

In order to test the actuators under real life conditions, they will be installed on a Wind Evaluation Breadboard (WEB). WEB is a dummy telescope, composed of seven flat segments. One segment is fixed (reference panel) and six segments are actuated. Three segments are representative for glass mirrors and three segments are representative for SiC mirrors. The WEB will be exposed to high frequency wind disturbances on a representative observatory site (La Palma, Canary Islands, Spain). WEB will test the performance of the control systems, actuators and structure at system level. The test program will start in the second half of 2008.

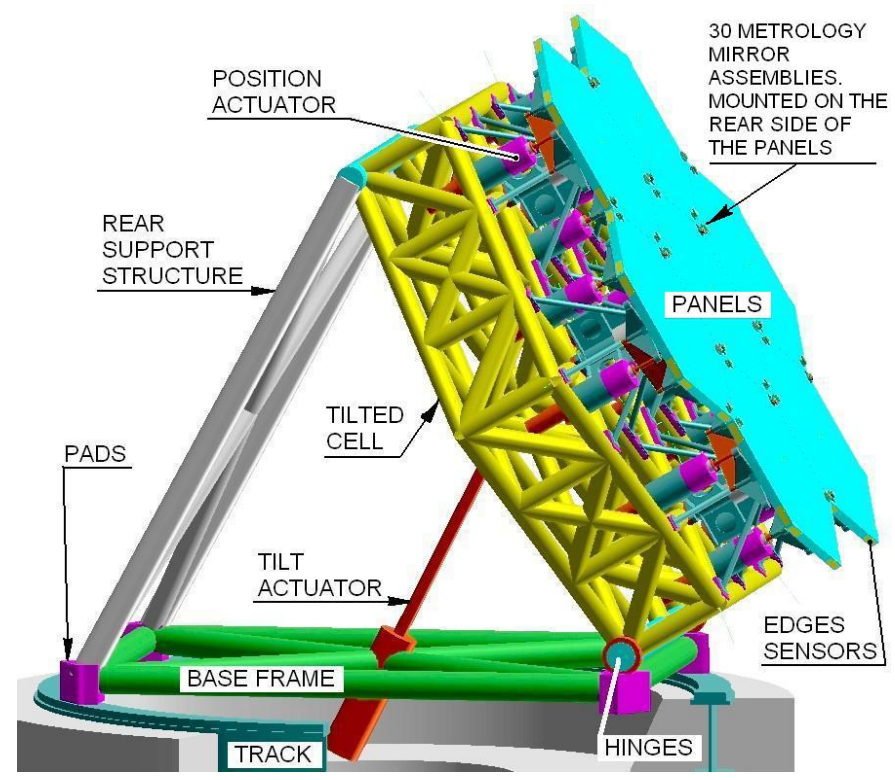

Figure 9 ESO Wind Experiment Breadboard (image courtesy of ESO) 


\section{CONCLUSIONS}

All PACT actuators have been built, and delivered to ESO for implementation in the WEB.

From the verification program it can be concluded that the PACT actuator meet all E-ELT requirements (except the power dissipation for the glass segment actuator). This is caused by the holding current of the stepper motor. A different motor will be used for the final E-ELT actuators, if the glass segments are the selected baseline.

The control system is optimized to work with a low bandwidth, which provides extra margin at system level (e.g. with respect to structural stiffness and edge sensors).

TNO has developed an actuator for piston and tip/tilt control of large segmented mirrors. The actuator can be used in EELT but the design is scalable and could be adapted to enable implementation in other telescope systems as well.

The actuator uses standard industrial components and can be built at relatively low cost.

\section{ACKNOWLEDGEMENT}

The TNO team wishes to acknowledge the work of Eduard van der Merché, who sadly passed away in April 2007. Not only did Eduard make a vital contribution to the control algorithms, but we will also miss him as a colleague and friend.

\section{REFERENCES}

1. "The W.M. Keck Telescope segmented primary mirror active control system," R. C. Jared et al; SPIE Vol. 1236 Advanced Technology Optical Telescopes IV, pp. 996-1008, 1990

2. "Design and preliminary test of a precision segment positioning actuator for the California Extremely Large Telescope primary mirror", Kenneth R. Lorell et al; SPIE Vol. 4840 Future Giant Telescopes, 2003

3. "Active mirror support using pneumatic actuators", Richard J. Bennet et al; SPIE Vol. 5497 Modeling and Systems Engineering for Astronomy, 2004

4. "OWL opto-mechanics, phase A”, E. Brunetto, SPIE Vol. 5489 Ground based telescopes, 2004 